\title{
Radioablação de tumores hepáticos primários e metastáticos. Onde estamos e para onde vamos?
}

\author{
Radiofrequency ablation of primary and metastatic liver tumors. Where \\ are we and where we are going?
}

Marcelo A. F. Ribeiro Jr. ${ }^{1}$, Francine Papaiordanou², Marina Epstein ${ }^{3}$, Alexandre Zankenko Fonseca ${ }^{4}$, Eleazar Chaib ${ }^{5}$

$\mathrm{O}$ carcinoma hepatocelular (HCC) é o tu mor sólido mais comum no mundo, com incidência estimada em um milhão de novos pacientes por ano. Além disso, atrás apenas dos linfonodos, o fígado é o segundo maior alvo de metástases provenientes de outros tumores sólidos, o que é particularmente comum em pacientes com adenocarcinoma coloretal, por tratar-se do órgão mais comumente acometido na doença metastática neste tipo de neoplasia. Infelizmente, apenas 5 a $15 \%$ dos novos pacientes diagnosticados com HCC ou metástase hepática de câncer coloretal são submetidos a uma ressecção curativa, o que ainda é considerado o "padrão ouro" para o tratamento desses tumores. Pacientes com doença confinada ao fígado podem não ser candidatos à ressecção devido a lesões multifocais, proximidade do tumor de ramos vasculares nobres ou a estruturas biliares que impeçam uma ressecção com margens seguras, biologia tumoral potencialmente desfavorável, como por exemplo, a presença de mais de quatro metástases hepáticas ou pacientes portadores de reserva funcional hepática inadequada, relacionada à coexistência de cirrose ${ }^{1}$. Nesses casos, uma das formas de tratamento alternativo é a ablação por radiofrequiência (RFA), que vem sendo amplamente utilizada devido a seus potenciais benefícios, incluindo redução da mortalidade e morbidade ${ }^{2}$.

Pode ser aplicada por via percutânea, laparoscópica ou por meio de laparotomia como forma de tratamento primário ou como coadjuvante ao trata-
1. Professor Titular da Disciplina de Cirurgia Geral, Chefe do Núcleo de Clínica Cirúrgica e Coordenador do Curso de Medicina da Universidade de Santo Amaro - UNISA. Professor de Habilidades Cirúrgicas da Universidade Cidade de São Paulo UNICID.

2. Acadêmica do $6^{\circ}$ ano do Curso de Medicina da Universidade Cidade de São Paulo - UNICID.

3. Residente do Programa de Cirurgia Geral da Universidade de Santo Amaro - UNISA.

4. Professor Assistente da Disciplina de Cirurgia Geral da Universidade de Santo Amaro - UNISA.

5. Livre Docente e Diretor do Laboratório de Investigação Médica da Disciplina de Transplante e Cirurgia do Fígado do Departamento de Gastroenterologia da Faculdade de Medicina da Universidade de São Paulo. Former Honorary Registrar Department of Surgery, Addenbrookes Hospital, University of Cambridge, England, UK.
Correspondência: Prof. Dr. Marcelo A. F. Ribeiro Jr. Rua Nova York, 609 apto 122B São Paulo - SP 04560-001 mribeiro@cwaynet.com.br

Artigo recebido em 02/12/2009 Aprovado em 18/12/2009 
mento operatório. Na via aberta tem-se um maior controle assim como exposição das estruturas abdominais, o que permite um rastreamento e posicionamento dos probes de maneira adequada assim como afastamento de estruturas adjacentes. Desta forma a via percutânea fica reservada aos pacientes não candidatos ao tratamento cirúrgico, ou nos casos de recorrência tumoral. Estes podem ser tratados repetidas vezes sem efeitos cumulativos, sendo a aplicação feita à demanda, conforme o surgimento de novas lesões $^{3}$.

Quanto aos hepatocarcinomas, em um estudo recente de Massarweh et al (2010) ${ }^{4}$ onde foi realizado um levantamento entre 1998 e 2005 no banco de dados Norte Americano de sobrevida e resultados de tratamentos, foram avaliados 22.103 pacientes portadores de HCC e maiores de 18 anos. Foram estudadas as modalidades de tratamento oferecidas (RFA, transplante ou ressecção) e as sobrevidas de 1 e 2 anos. Destes, 4.924 (22\%) pacientes foram submetidos a alguma forma de tratamento, sendo que a RFA representou a modalidade terapêutica que mais ampliou sua aplicação no período de 8 anos, com um aumento de $43 \%$. Convém salientar, entretanto que quando se comparou a sobrevida de 1 e 2 anos nos grupos acima, nos anos de 1998, 2002 e 2004, o único que apresentou significância estatística de aumento da sobrevida foram os pacientes submetidos à ressecção. Uma observação interessante diz respeito ao aumento progressivo na utilização de métodos ablativos em geral assim como RFA no tratamento de tais lesões, por exemplo, a porcentagem de utilização em 1998 e 2005 expressa em porcentagem por cada 100 pacientes com HCC relatada foi $3,2 \%$ e $12,1 \%$ no grupo de métodos ablativos total e $0,4 \%$ para $6,2 \%$ no grupo RFA, ambos com $\mathrm{P}<0.001$. Outro tema de discussão entre os especialistas da área versa sobre os resultados comparativos entre RFA e ressecção de HCC menores do que $5 \mathrm{~cm}$. Chen et al (2006) $)^{5}$, publicaram os resultados de estudo prospectivo comparando as duas modalidades de tratamento de maneira prospectiva e randomizada. Para tanto foram selecionados inicialmente 90 pacientes para cada braço do estudo, sendo que, 71 pacientes efetivamente receberam RFA e 90 foram submetidos à ressecção. Destes em 69 pacientes foi removido um único segmento hepático, em 16 casos foram removidos dois segmentos e em 3 casos foram três ou mais segmentos. Foram observados quanto à sobrevida global após 1, 2,3 e 4 anos para casos de RFA e ressecção os seguintes resulta- dos: $95,8 \%, 82,1 \%, 71,4 \%, 67,9 \%$ e $93,3 \%, 82,3 \%$, $73,4 \%, 64 \%$ respectivamente. Não se observou diferença estatística na sobrevida livre de doença, entretanto as complicações foram mais frequientes e mais graves no grupo submetido a cirurgias (50 de 90 operados vs. 3 de 71 RFA, $\mathrm{P}<0.05$ ). Guo et al ${ }^{6}$ realizaram um estudo comparativo multicêntrico não randomizado entre pacientes tratados por RFA percutânea versus hepatectomias parciais, as lesões não ultrapassaram um número total de três tumores por paciente e tamanho máximo de 5 centímetros. Foram incluídos no grupo RFA 86 pacientes e no grupo de ressecção 73 casos. Foi observada uma maior taxa de complicações (19,2 vs. $8,1 \%)$ assim como tempo de permanência hospitalar (9 vs. 3 dias) no grupo de ressecções. A sobrevida global após 1, 3 e 5 anos no grupo ressecção foi 91,8, 68,7 e 44,5\% e no grupo RFA 94,2, 64,4 e $21,2 \%$ respectivamente. Quanto a sobrevida livre de doença para os dois grupos observou-se 62,1, 33,6 e $3,6 \%$ e $29,4,2,7$ e $0 \%$, respectivamente. Observou-se significância estatística para ambas as análises foram favoráveis ao grupo de ressecção, concluindo que no grupo submetido as ressecções há uma melhor sobrevida global, entretanto o grupo de pacientes submetidos a RFA se beneficiam das menores taxas de complicações e menor tempo de permanência hospitalar.

Livraghi et al (2008) ${ }^{7}$ descreveram em estudo multicêntrico prospectivo uma série de 218 pacientes portadores de $\mathrm{HCC}$ menores do que $2 \mathrm{~cm}$, únicos classificados como sendo muito precoces ou T1 conforme classificação do grupo de estudos de câncer de fígado de Barcelona tratados por via percutânea. Foram avaliados de resposta local sustentada e completa assim como complicações relacionadas ao método e sobrevida em 5 anos dos 100 pacientes onde as lesões foram consideradas potencialmente ressecáveis. A média de seguimento foi de 31 meses. Quanto a resposta completa e sustentada após a RFA, a mesma foi observada em $97,2 \%$ dos casos e foi observada falha no tratamento em 2,7\%. Dos 100 casos potencialmente ressecáveis que foram tratados por meio da RFA, a sobrevida em 3 e 5 anos foi estatisticamente superior ao grupo de 118 casos não candidatos a ressecção, $89 \%$ e $68 \%$ vs. $75 \%$ e $47 \%(P=0.013)$. $\mathrm{Na}$ análise univariada os fatores idade, surgimento de novas lesões e ressecabilidade apresentaram significância estatística. Na análise multivariada, observouse apenas o surgimento de novas lesões apresentou tal significância. Frente a tais dados os autores suge- 
rem que em lesões d" $2 \mathrm{~cm}$ a RFA pode ser considerada o tratamento de eleição mesmo quando a ressecção seja factível, podendo-se inclusive combinar outras modalidades de tratamento nos casos onde a RFA tenha falhado.

Quanto às metástases coloretais, As taxas de recorrência local após ressecção R0 (microscopicamente negativos) hoje se situa em torno de $5 \%$ e a sobrevida em 5 anos varia entre $35 \%$ e $55 \%$. Em contraste, a eficácia da RFA apresenta números heterogêneos. Alguns estudos apresentam taxas de recorrência local de $10 \%$ enquanto outros apresentam valores da ordem de 40-50\%. Quanto a sobrevida, os números também são algo conflitantes, com números que variam entre $55 \%$ em 3 anos a $30 \%$ em 5 anos, valores estes encorajadores ${ }^{8}$. A RFA vem sendo amplamente aplicada nos casos de pacientes não candidatos a ressecção podendo com isto oferecer destruição do tumor e aumento da expectativa de vida, entretanto, a recorrência peri-lesional ocorre em aproximadamente 9 a $18 \%$ dos casos $^{9-12}$. Um dos primeiros estudos avaliando a aplicação da RFA em metástases foi realizado por Lencioni et al ${ }^{13} \mathrm{em} 1998$, onde realizaram a aplicação da mesma em 29 pacientes não candidatos a ressecção. Consideraram o resultado satisfatório em $77 \%$ dos casos, sendo que nas lesões com menos de $3 \mathrm{~cm}$ a efetividade atingiu $87 \%$ dos casos, entretanto com um seguimento médio de apenas 3 a 9 meses. Siperstein et al (2007) ${ }^{9}$ descreveram a serie com maior tempo de seguimento para MCR tratadas por meio da RFA. Foram avaliados 234 pacientes submetidos a 292 RFA, com uma média de 2,8 lesões por caso e um tamanho médio de $3,9 \mathrm{~cm}$. Os seguintes fatores apresentaram impacto no resultado: pacientes com $\leq 3$ lesões versus $>3$ lesões $(\mathrm{P}=$ $0.0018)$; tamanho $\leq 3 \mathrm{~cm}$ versus $>3 \mathrm{~cm}(\mathrm{P}=0.07)$, dosagem de CEA $\leq 200 \mathrm{ng} / \mathrm{mL}$ versus $>200 \mathrm{ng} / \mathrm{mL}$ $(\mathrm{P}=0.003)$. A presença de doença extra-hepática assim como tipo de quimioterapia não influenciaram nos resultados na sobrevida média. Pacientes que apresentaram falha no tratamento quimioterápico novos regimes de tratamento não beneficiaram os pacientes, sendo a sobrevida atuarial após 5 anos para este grupo foi de $18,4 \%$ nos casos que receberam a RFA versus zero para os que receberam apenas a quimioterapia. Em casos onde há o aumento do tamanho das lesões, assim como a aplicação por via percutânea fazem com que as taxas de recidiva possam chegar a atingir $60 \%$ dos $\operatorname{casos}^{11}$.
Considerando-se que as ressecções hepáticas diminuem a quantidade de parênquima remanescente com risco de surgimento de novas metástases, Abdalla et al (2004) ${ }^{10}$ realizaram um estudo com achados interessantes quanto ao surgimento de novas lesões. Foram acompanhados 190 pacientes submetidos cirurgia para ressecção com intenção curativa sendo que em 158 a ressecção completa não foi possível (101 receberam RFA associada à ressecção e 57 somente RFA). Dentre os que receberam exclusivamente RFA, 44\% apresentaram recorrência hepática exclusiva confinada ao fígado (novas lesões e recorrências verdadeiras), mas apenas em $9 \%$ a recorrência foi verdadeira. Pode-se então inferir que nos outros $35 \%$ dos pacientes as lesões foram consideradas como novas lesões. Em contraste, apenas 11\% dos pacientes submetidos à ressecção com escopo curativo apresentaram recorrência no parênquima. Sendo que apenas $2 \%$ apresentaram recorrência verdadeira, sugerindo, portanto que em $9 \%$ houve o surgimento de novas lesões. Entretanto, apesar da RFA ser menos efetiva em termos de controle local (9\% versus $2 \%$ em ressecções), um grande benefício da ressecção hepática talvez seja um menor surgimento de novas lesões no fígado. Otto et al (2010) ${ }^{14}$ em estudo recente descrevem o primeiro trabalho da literatura onde o grupo RFA poderia ser submetido à ressecção cirúrgica, não sendo, portanto um tratamento aos pacientes não candidatos a ressecção, onde a RFA foi utilizada como tratamento de primeira linha. Neste estudo os casos encaminhados a ressecção foram aqueles onde o tamanho da lesão excedia $5 \mathrm{~cm}$ ou onde as localizações não permitiam a ablação. Outro dado relevante diz respeito à característica da amostra a qual foi classificada como pacientes portadores de metástases precoces, ou seja, lesões identificadas nos primeiros doze meses apos a cirurgia para remoção do tumor do cólon. Quanto à amostra os casos tratados por meio de RFA ( $\mathrm{N}=28)$ apresentavam diâmetro máximo de $5 \mathrm{~cm}$ e não mais do que 5 lesões. As características dos grupos RFA e ressecção $(\mathrm{N}=82)$ foram as mesmas a exceção do tamanho conforme explicitado acima. Quanto aos resultados obtidos, a taxa de recorrência local foi de $32 \%$ no grupo RFA e $4 \%$ no grupo ressecção $(\mathrm{P}<0.001)$, no tocante as recorrências sistêmicas, foi observado $32 \%$ e $37 \%$ respectivamente $(\mathrm{P}=0,820)$. $\mathrm{O}$ tempo de progressão da doença foi significativamente menor no grupo RFA (203 vs 416 dias; $\mathrm{P}=0.017$ ). Observou-se que após o 
tratamento primário 9 pacientes do grupo RFA e 8 do grupo ressecção foram candidatos a novos tratamentos por uma ou outra forma de tratamento, resultando em valores idênticos apos 3 anos de sobrevida livre de doença assim como de sobrevida global em ambos os grupos: $67 \%$ e $60 \%$ respectivamente $(\mathrm{P}=0.93)$. Foi observado pelos autores que pacientes submetidos a cirurgia apresentavam maiores tempos de permanência hospitalar (13 vs 2 dias em média). Quanto à recorrência, no total 20 dos 28 casos do grupo RFA (71\%) e somente 39 dos 82 casos ressecados (48\%) apresentaram recorrência tumoral, convém salientar que os grupos não diferiram quanto a estádio TNM, tempo até o tratamento das metástases, tamanho e numero de lesões, valores de CEA ou presença de doença uni ou bilobar. Com isto concluem que quando comparado a ressecção o tratamento com RFA é menos radical do que a cirurgia.

Portanto frente São dados da literatura atuais, a radioablação de tumores tem hoje um papel bem definido para pacientes portadores de HCC, com lesões menores do que 3 centímetros, que possam ser tratadas preferencialmente por via percutânea, principalmente para pacientes em lista para transplante ou para ser utilizada em conjunto a ressecção por via aberta. Os resultados para este grupo são bastante animadores e a seleção correta dos casos o fator determinante para o sucesso terapêutico. Já nos casos de metástase de tumores coloretais os resultados apontam taxas de sobrevida e recorrência muito superiores para os pacientes operados quando comparados aos casos tratados por meio de RFA, ficando esta modalidade terapêutica reservada para os casos onde a cirurgia não seja factível, para tratamento de pequenas lesões em conjunto a ressecção, não devendo, portanto ser considerada uma modalidade de tratamento capaz de substituir o tratamento operatório. Acredito que em futuro próximo teremos a radioablação como modalidade técnica realizada pelos radiologistas, ficando reservado aos cirurgiões de fígado a utilização das microondas que apresentam resultados até o momento animadores e que podem inclusive ampliar as indicações dos métodos ablativos por via aberta.

\section{REFERÊNCIAS}

1. Pearson AS, Izzo F, Fleming RY, Ellis LM, Delrio $P$ and Roh MS. Intraoperative radiofrequency ablation or cryoablation for hepatic malignancies. Am J Surg 1999; 178:592-9.

2. Livraghi T, Solbiati L, Meloni MF, Gazelle GS, Halpern EF, Goldberg SN. Treatment of focal liver tumors with percutaneous radio-frequency ablation: complications encountered in a multicenter study. Radiology 2003; 226:441-51.

3. Ribeiro JR MAF,Gama Rodrigues JJ, Habr-Gama A, Chaib E, D'Ippolito G, Fonseca AZ, Saad Jr WA, Saad WA. Radiofrequency ablation of primary and metastatic liver tumors-the 4 years experience. Hepatogastroenterology 2007; 54:1170-1175.

4. Massarweh NN, Park JO, Farjah F, Yeung RSW, Symons RG, Vaughan TL, Baldwin LM, Flum DR. Trends in the utilization and impact of radiofrequency ablation for hepatocellular carcinoma. J Am Col Surg 2010; 210:441-448.

5. Chen MS, Li JQ, Zheng Y, Guo RP, Liang HH, Zhang YQ, Lin XJ, Lau WY. A prospective randomized trial comparing percutaneous local ablative therapy and partical hepatectomy for small hepatocellular carcinoma. Ann Surg 2006; 243:321328.

6. Guo WX, Zhai B, Lai ECH, Li N, Shi J, Lau WY, Wu MC, Cheng $S Q$ - Percutaneous radiofrequency ablation versus partial hepatectomy for multicentric small hepatocellular carcinomas: A nonrandomized comaprative study. World J Surg 2010; 34:2671-2676.

7. Livraghi T, Meloni F, Di Stasi M, Rolle E, Solbiati L, Tinelli C, Rossi S. Sustained complete response and complications rates after radiofrequency ablation of very early hepatocelIular carcinoma in cirrhosis: is resection still the treatment of choice? Hepatology 2008;47:82-89.

8. Hurwitz H, Fehrenbacher L, Novotny W, Cartwright T, Hainsworht J, Heim W et al. Bevacizumab plus irinotecan, fluorouacil and leucovorin for metastatic colorectal cancer. N Engl J Med 2004;350:2335-2342.

9. Siperstein AE, Berber E, Ballem N, Rikesh T, Parikh RT. Survival after radiofrequency ablation of colorectal liver metastases: 10-year experience. Ann Surg 2007;246:559567.

10. Abdalla EK, Vauthey JN, Ellis LM, Ellis V, Pollock R, Broglio KR, Hess K, Curley SA. Recurrence and outcomes following hepatic resection, radiofrequency ablation, and combined resection/ablation for colorectal liver metastases. Ann Surg 2004;239:818-825

11. Mulier S, Ni Y, Jamart J, Ruers T, Marchal G, Michel L. Local recurrence after hepatic radiofrequency coagulation: Multivariate meta-analysis and review of contributing factors. Ann Surg 2005;242:158-171.

12. Mulier S, Ruers T, Jamart J, Michel L Marchal G, Ni Y Radiofrequency ablation versus resection for resectable colorectal liver metastases: time for a randomized trial? Ann Surg Oncol 2008;15:144-157.

13.Lencioni R, Goletti O, Armillotta N, Paolicchi A, Moretti M, Cioni D, Donatti F, Cicorelli A, Ricci S, Carrai M, Conte PF, Cavina E, Bartolozzi C. Radio-frequency thermal ablation of liver metastases with a cooled-tip electrode needle: results of a pilot clinical trial. Eur Radiol 1998; 8:1205-1211.

14. Otto G, Duber C, Hoppe-Lotichius M, Konig J, Heise M, Pitton MB. Radiofrequency ablation as first-line treatment in patients with early colorectal liver metastases amenable to surgery. Ann Surg; 2010; 251:796-803. 